\title{
Multiple primary malignant neoplasms - case report
}

\author{
Cornelia Amalinei ${ }^{1 *}$, Raluca Balan ${ }^{1}$, Luminita Ivan ${ }^{2}$, Razvan Socolov ${ }^{3}$, \\ Demetra Socolov ${ }^{3}$, Coriolan Cotutiu ${ }^{1}$ \\ 1 Pathology Department, \\ University of Medicine and Pharmacy Iasi, \\ Romania \\ 2 Pathology Laboratory, \\ Military Hospital Iasi, \\ Romania \\ 3 Obstetrics and Gynecology Department, \\ University of Medicine and Pharmacy Iasi, \\ Romania
}

Received 7 December 2006; accepted 6 February 2006

\begin{abstract}
The synchronous occurence of primary carcinomas of endometrium and ovary is well recognized. Malignant peripheral nerve sheath tumours (MPNSTs) may also rarely occur in patients diagnosed with malignancies of the female genital tract. We report a rare case of synchronous primary carcinomas of endometrium and ovary, followed by a metachronous retroperitoneal MPNST. Ascites cytology and endometrial biopsy, followed by hysterectomy and bilateral adnexectomy, were performed to remove the synchronous tumors. Histology was suggestive of synchronous endometrial endometrioid carcinoma and ovarian mucinous adenocarcinoma. After the removal of the retroperitoneal tumor, a MPNST was diagnosed by immunohistochemistry. The patient developed two consecutive vaginal tumors diagnosed as metastases of the previously diagnosed endometrial carcinoma. Although synchronous tumors of endometrium and ovary were relatively early staged and consequently had a favorable prognosis, subsequently occuring implants along the lower genital tract and the metachronous MPNST added up to a poor prognosis.

(c) Central European Science Journals Warsaw and Springer-Verlag Berlin Heidelberg. All rights reserved.
\end{abstract}

Keywords: Synchronous primary carcinomas of endometrium and ovary, Ovarian mucinous adenocarcinoma, Immunohistochemistry, Metastatic tumor, Malignant peripheral nerve sheath tumor (MPNST)

\footnotetext{
* E-mail: amalinei@umfiasi.ro
} 


\section{Introduction}

The synchronous occurrence of primary carcinomas of the endometrium and ovary is well recognized. Estrogen stimulation is often postulated to be a significant factor in the development of the endometrial carcinoma in such cases [1].

MPNST may also occur in patients diagnosed with malignancies of the female genital tract in cases of associations with Proteus Syndrome [12].

We report a rare association of synchronous primary carcinomas of endometrium and ovary, followed seven months later by a MPNST.

\section{Clinical history}

A 66 years-old female patient, having class II New York Heart Association (NYHA) heart failure, presented with uterine bleeding and ascites, in October 2002. Sonography showed an enlarged uterus, with a $10 \mathrm{~mm}$ thick endometrium and an ovarian mass over $20 \mathrm{~cm}$ in its largest diameter, with combined solid and cystic areas, and few inner Doppler's echoes. Ascites cytology revealed mucous secreting tumor cells. Endometrial carcinoma of endometrioid pattern was diagnosed on endometrial biopsy. Hysterectomy, bilateral adnexectomy and partial omentectomy were performed, followed by 6 lines of adjuvant chemotherapy, using Cisplatin combined with Cyclophosphamide. The clinical course was favorable.

Seven months after the initial diagnosis, the patient presented with a retroperitoneal, pararectal solid tumor mass. On magnetic resonance imaging (MRI), the tumor extended into the vaginal wall. A malignant peripheral nerve sheath tumor (MPNST) was diagnosed after surgical excision. Palliative radiotherapy, fractioned in 10 doses of 30 Gy, was performed as additional treatment.

One year after the surgery, the patient developed another tumor mass on the vaginal wall. An excision biopsy showed a metastatic adenocarcinoma corresponding to the previously diagnosed endometrioid carcinoma. Six months later another tumor developed on the vaginal wall and cytology revealed another metastasis of the endometrioid carcinoma. Both metastases were treated by combined chemotherapy and radiotherapy.

\section{Material and methods}

Peritoneal cytology was processed by centrifugation, followed by May Grünwald Giemsa (MGG) staining. Biopsy material and surgical specimens were routinely formalin fixed and paraffin embedded. All samples were routinely stained with HE, followed by PAS, Alcian Blue, and Gomori stainings to determine the histological diagnosis. Specimens from the tumor and the vaginal extension were routinely processed, stained with HE, van Gieson, and Alcian Blue. For immunohistochemistry, 4- $\mu \mathrm{m}$-thick sections were deparaffinized with toluene for $15 \mathrm{~min}$ and dehydrated through a series of graded alcohols. Endogenous peroxidase activity was blocked by incubation (10 min) in $0.3 \% \mathrm{H}_{2} \mathrm{O}_{2}$ in 
methanol. The sections were gently rinsed with Tris-buffered saline (TBS) and then incubated with monoclonal antibodies against SM-Actin (clone 1A ${ }_{4}$ Biogenex AM128-5M), S-100 (clone 15E $\mathrm{E}_{2} \mathrm{E}_{2}$ Biogenex AM058-5M), Vimentin (clone $\mathrm{V}_{9}$ Biogenex AM074-5M), Desmin (Monoclonal Mouse IgG [33] Biogenex AM072-5M), cytokeratin cocktail (Monoclonal Mouse IgG Cocktail Biogenex AM372-5M), and neuron-specific enolase (NSE) (Monoclonal Mouse $\operatorname{IgG}_{1}\left[\mathrm{MIG}-\mathrm{N}_{3}\right]$ Biogenex) for $30 \mathrm{~min}$ and then with the secondary antibody (Multilink) for 20 min using Automatic OptiMax+ (Biogenex). The signal was detected using the streptavidin-biotin-peroxidase complex Label method (LSAB, StreptAviden), according to the manufacturer's recommendation (Biogenex). DAB (3,3' diaminobenzidine hydrochloride containing $0.08 \%$ hydrogen peroxidase) was used as a chromogen.

Immunohistochemical reactions were scored according the following categories: definitely negative when tumor cells did not stain, weak positive when not more than 10 $\%$ of the tumor cells were stained, focally positive when only scattered positivity was observed, moderately positive when 10-50\% of the neoplastic cells were stained, and strongly positivity when more than $50 \%$ of the tumor cells were stained.

\section{Pathologic findings}

On peritoneal cytology and endometrial biopsy, the diagnosis of a synchronous endometrioid carcinoma, G1, associated with an ovarian mucinous adenocarcinoma, G1, was made. Hysterectomy and bilateral adnexectomy were performed.

The uterus was enlarged and contained a single exophytic mass, with a firm consistency, a white section surface, and deep extension into the underlying myometrium. Histologically, an endometrioid adenocarcinoma was diagnosed. It contained glands resembling those of the normal endometrium (Fig. 1), lined by simple to pseudostratified or stratified columnar cells. A part of the tumor exhibited villous fronds containing delicate central cores. These areas of villoglandular aspect admixed with typical endometrioid areas did not modify the histologic diagnosis, as they comprised only a minor portion of the tumor. Myometrial invasion was more than one half of the myometrium. Neither solid nests of cells nor bizarre nuclear atypia were noted. Differential diagnosis was made with atypical complex hyperplasia, but irregular infiltration of glands associated with an altered fibroblastic stroma and the confluent glandular pattern with individual glands uninterrupted by stroma favored adenocarcinoma. Mucinous variant was excluded by the absence of intracytoplasmic mucin and confirmed by negative mucin stainings (PAS and Alcian Blue), except for small amounts of intraluminal mucin. Mixed carcinoma showing a combination of endometrioid and serous or clear cell adenocarcinoma showing a papillary pattern was excluded as it usually exhibits more complex papillary architecture and high-grade nuclear features. No cervical involvement (surface glandular or stromal) was noted.

The ovarian tumor presented as a large, unilateral, smooth surfaced, multilocular cystic mass (over $20 \mathrm{~cm}$ in its largest diameter), containing viscous mucoid material, 


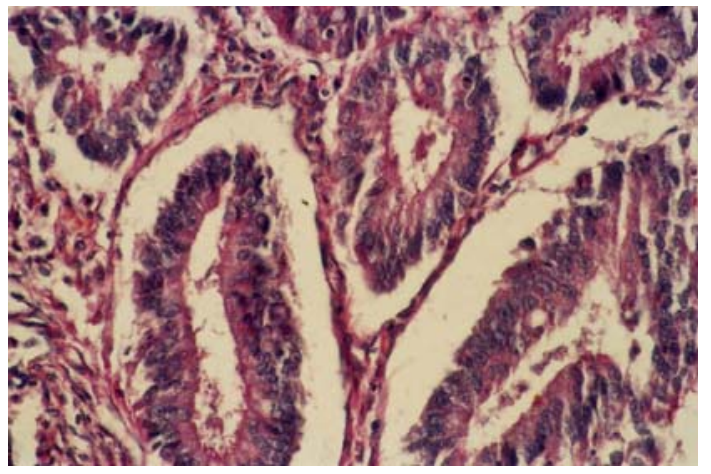

a)

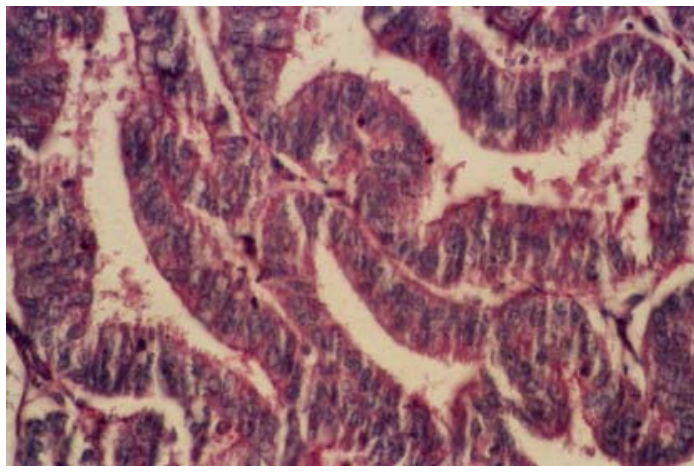

b)

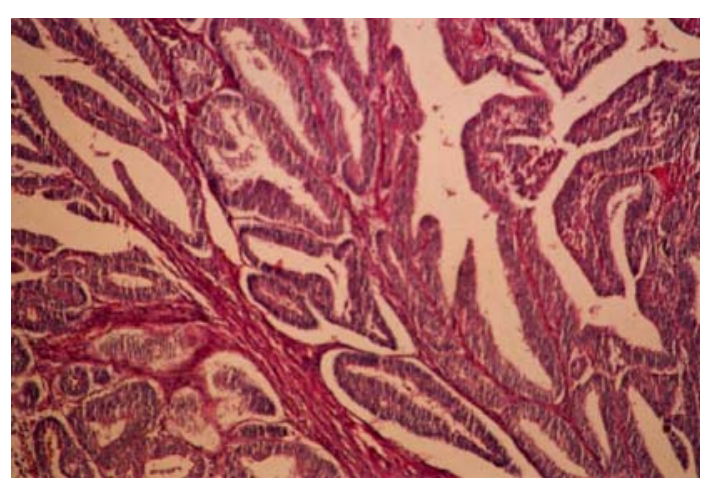

c)

Fig. 1 a) Endometrial endometrioid adenocarcinoma- well differentiated pattern (PAS $\times$ 40)

b) Endometrial endometrioid adenocarcinoma- architectural grade $1(\mathrm{PAS} \times 40)$

c) Endometrial endometrioid adenocarcinoma- villoglandular area $(\mathrm{PAS} \times 20)$

exhibiting hemorrhagic, necrotic, and papillary areas. Microscopy revealed complex papillary areas and "back-to-back" glands lined by malignant-appearing cells with little or no intervening stroma (Fig. 2). Stromal invasion was obvious, as tumoral areas larger than $10 \mathrm{~mm}^{2}$ and more than $3 \mathrm{~mm}$ in each of two linear dimensions were noted, showing infiltrative glands, tubules, cords, and cell nests. Positive peritoneal fluid cytology raised the stage to IC. Differential diagnosis was made with metastatic intestinal, pancreatic, biliary tract, gastric, or cervical mucinous carcinoma [5]. Primary rather than metastatic tumor was considered due to the following aspects: unilaterality, large size, smooth surface, expansile pattern of growth, and the complex papillary feature. Metastatic tumors were excluded due to the absence of the following criteria [6]: ovarian bilaterality, macroscopically friable aspect of the ovarian tumor, ovarian multinodular growth pattern, surface ovarian implants and vascular space invasion in ovary and corpus uteri.

As they were concomitant, neither being metastatic from the other, both carcinomas were considered as T1c, using TNM system of classification [13].

The metachronous retroperitoneal tumor presented as a poorly defined mass invading adjacent soft tissues, with a maximum diameter of $9 \mathrm{~cm}$, a pink-yellowish color, a convo- 


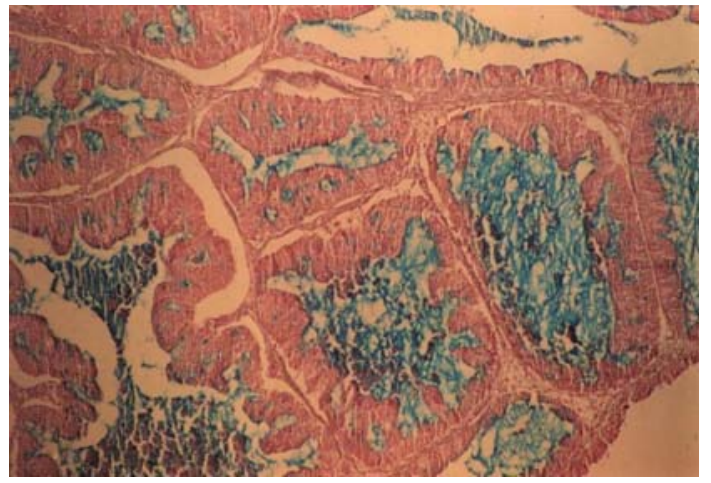

a)

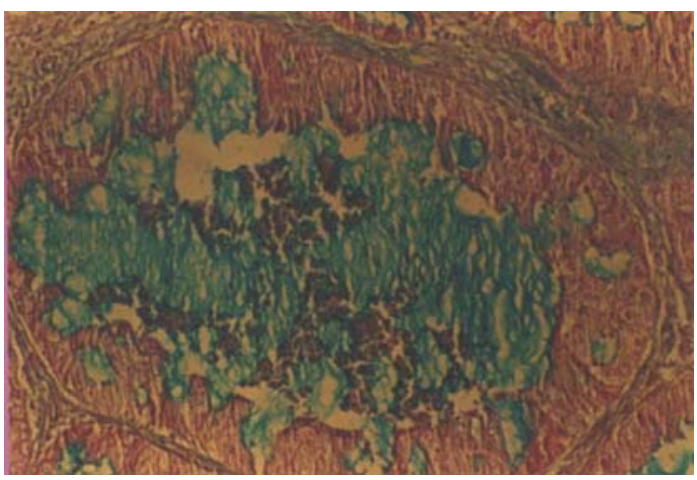

b)

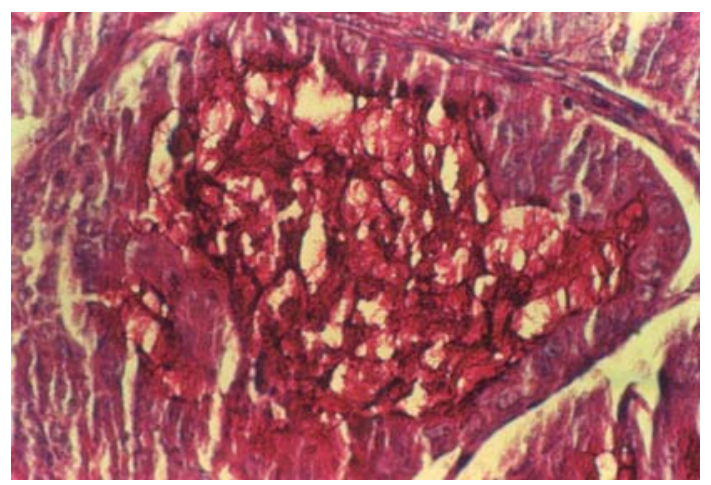

c)

Fig. 2 a) Ovarian mucinous adenocarcinoma synchronous with endometrial carcinoma $($ Alcian Blue $\times 10)$

b) Ovarian mucinous adenocarcinoma- intracellular and mostly extracellular mucin (Alcian Blue $\times 40)$

c) Ovarian mucinous adenocarcinoma $(\mathrm{PAS} \times 40)$

luted contour, containing hemorrhagic and necrotic areas. On microscopic examination, patterns reminiscent of fibrosarcoma or malignant fibrous histiocytoma were found. In other areas, the tumor cells resembled Schwann cells, with elongated nuclei and prominent bipolar processes. Rare fascicle formation was present. Other aspects were frequent mitoses and extreme nuclear anaplasia. Surgical margins were invaded by tumor cells. The immunoreactivity for cytokeratin cocktail was negative, for Vimentin was strongly positive, for S-100 protein was focally positive, for Desmin was negative, for SM-Actin was negative, and for NSE was strongly positive. Diagnosis was G III MPNST, stage III (Fig. 3). An undifferentiated carcinoma was excluded because of negative cytokeratin immunoreactivity and fibrosarcoma was excluded because of S-100 protein expression. The main differential diagnosis was that with a myxoid liposarcoma. As in MPNST, in liposarcoma both Vimentin and S-100 protein are positive, but liposarcoma was excluded due to the positivity of NSE (observed only in MPNST), associated with SM-Actin and Desmin negativity.

The vaginal tumors were diagnosed as metastatic adenocarcinoma resembling the 


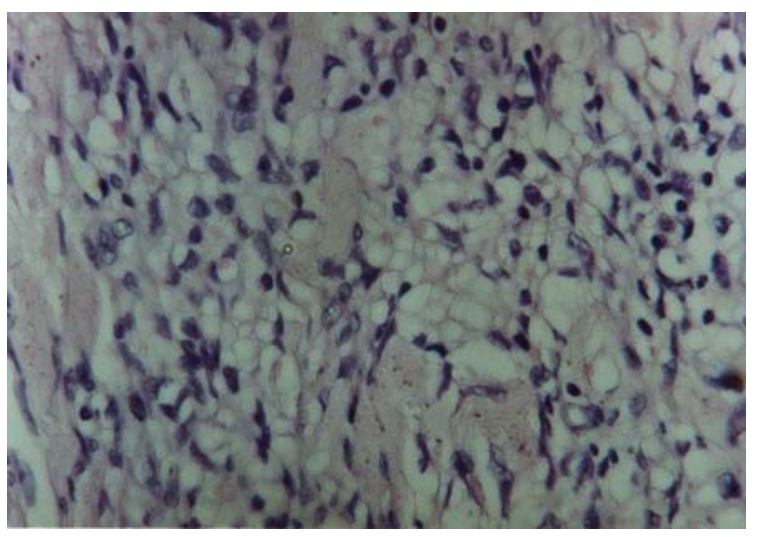

a)

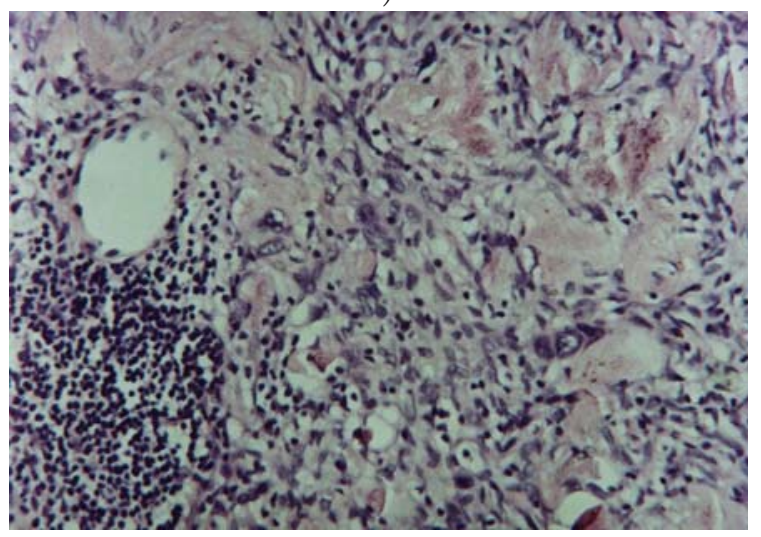

c)

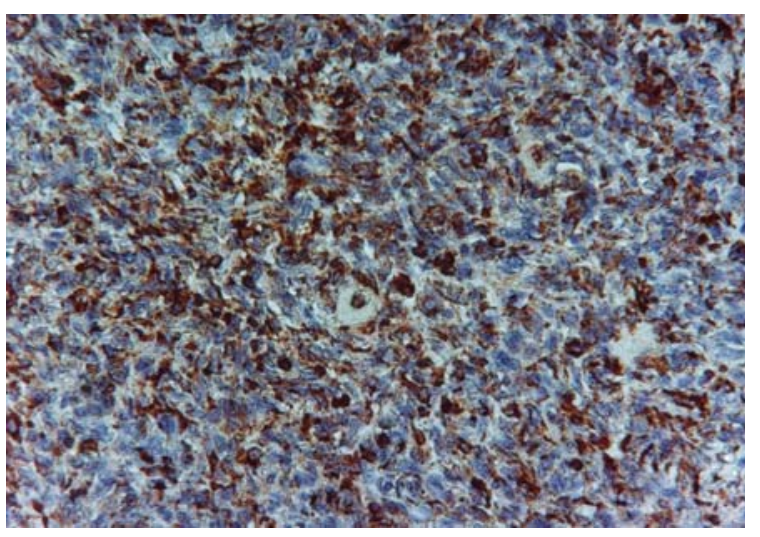

e)

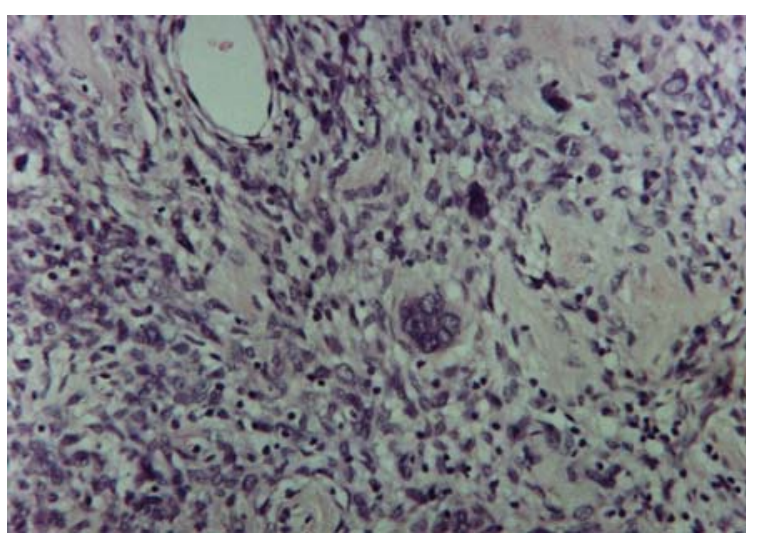

b)

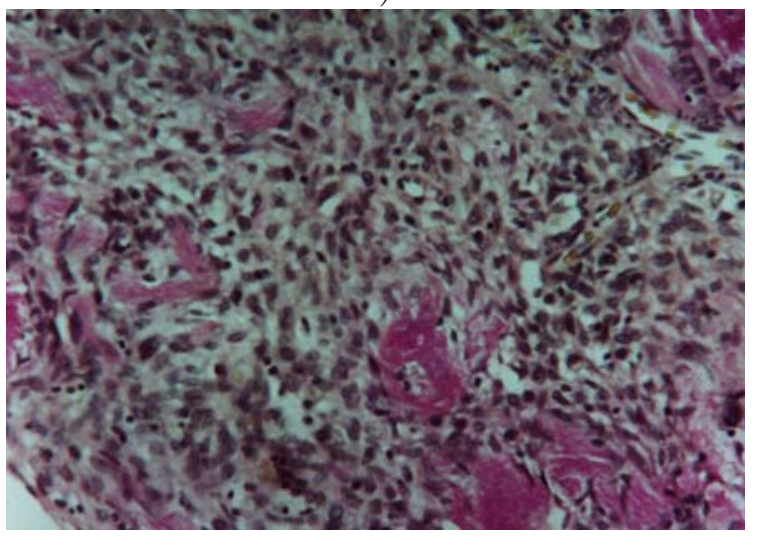

d)

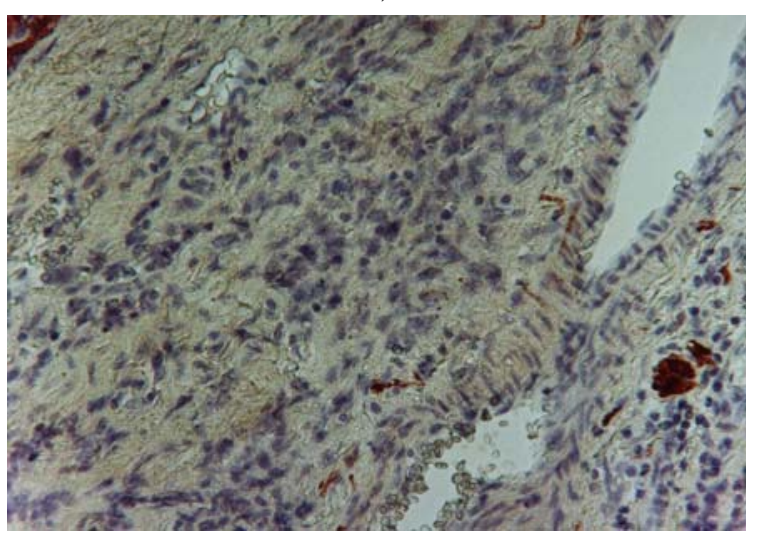

f)

Fig. 3 a) Area of MPNST with myxoid pattern $(\mathrm{HE} \times 40)$

b) Area of MPNST with giant cells $(\mathrm{HE} \times 20)$

c) Area of MPNST with myxoid pattern and lymphoid aggregate $(\mathrm{HE} \times 20)$

d) MPNST- evidence of collagen component (van Gieson $\times 40$ )

e) IHC positivity for Vimentin in MPNST $(\times 20)$

f) IHC positivity for S-100 in MPNST- focal positivity $(\times 20)$ 


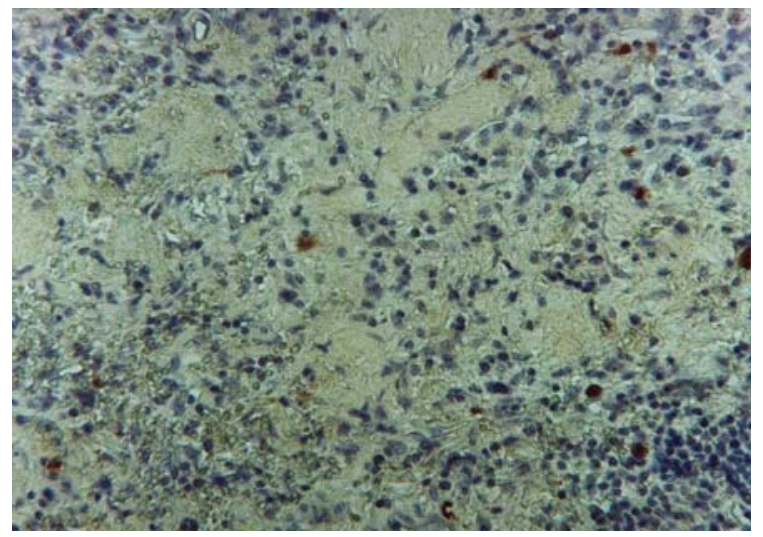

g)

Fig. 3 (continued) g) IHC positivity for S-100 in MPNST- scattered positive cells ( $\times$ 10)

previously diagnosed endometrioid carcinoma (Fig. 4).

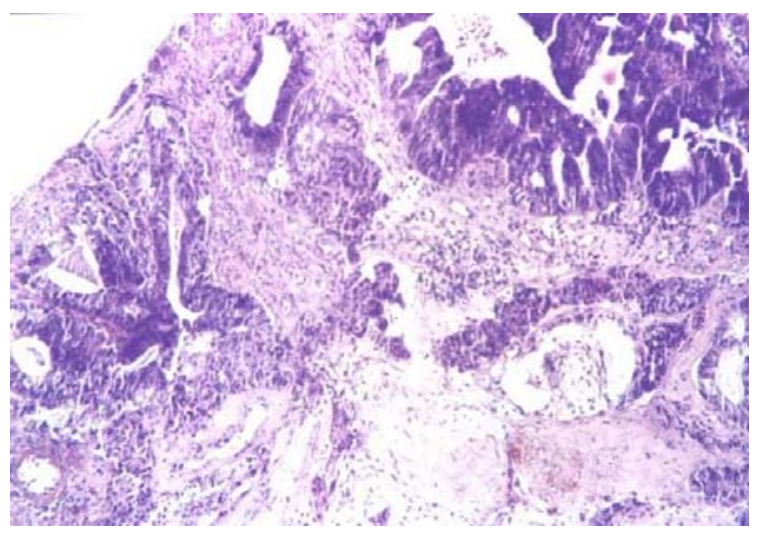

a)

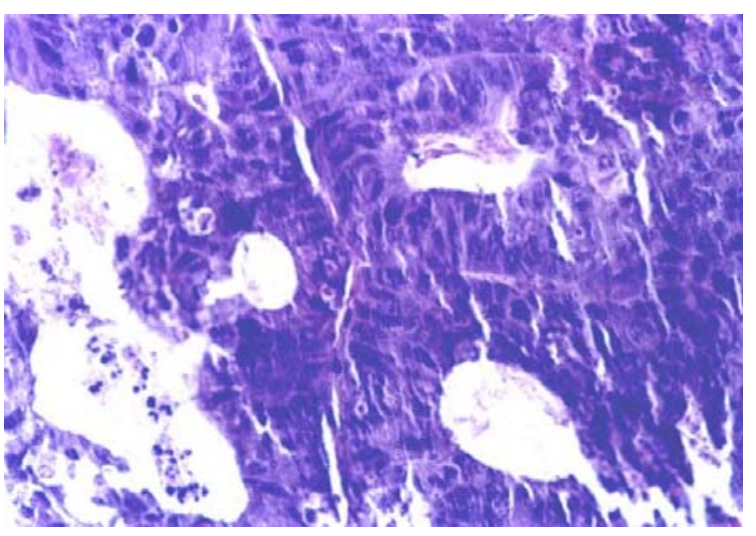

b)

Fig. 4 a) Vaginal metastasis of endometrioid carcinoma showing also some papillary areas $(\mathrm{HE} \times 10)$

b) Vaginal metastasis of endometrioid carcinoma $(\mathrm{HE} \times 20)$

\section{Discussions}

The surface epithelium of the ovary has the same embryologic derivation as the Mullerian duct and therefore, in adults, a given carcinogenic stimulus may produce similar epithelial proliferations in both structures. Cancers developing concomitantly in these locations are not infrequently misdiagnosed as metastatic tumors [14, 15]. However, the overall survival of these patients suggests multifocal rather than metastatic disease [14]. These tumors should be separated from true metastatic carcinomas of the female genital tract, since each of these two categories carries a different prognosis and clinical implications. In some cases, however, it may be difficult to distinguish between metastatic 
and independent malignancies because the tumors may share some features, so immunohistochemistry, flow cytometry, and molecular pathology techniques may be necessary. Histological dissimilarity of the tumors, limited myometrial invasion of the endometrial tumor, lack of vascular invasion of both tumors, unilaterality of the ovarian tumor, and central localization of the ovarian tumor without surface implants in the present case were highly suggestive of independent primaries.

Endometrioid adenocarcinomas begin as monoclonal outgrowths of genetically altered premalignant cells, and many bear genetic stigmata of microsatellite instability, KRAS mutation and loss of PTEN (phosphatase and tensin homologue deleted on chromosome 10) function that are conserved in subsequent cancers [7]. PTEN checks cell division and enables apoptosis through a phosphoinositol-3-kinase-Akt pathway. Functional consequences of PTEN mutation may be modulated in part by the hormonal environment, as PTEN is expressed only during the estrogen-driven proliferative phase of the endometrium. Loss of PTEN function occurs as an early event that may precede cytological and architectural changes, but one-third to one-half of endometrial adenocarcinomas type I (endometrioid) continue to express PTEN protein, so the value of PTEN immunohistochemistry is limited $[10,11]$. Other alterations were observed in estrogen receptor (ER), progesterone receptor (PR), $\beta$-catenin, cyclin D1, p53 (much lower than in type II endometrial carcinoma), KRAS, MLH1, p27 (lower than in type II endometrial carcinoma), p16, Bcl-2 and Bax (the last two almost equal with those of type II endometrial carcinoma) $[10,11]$.

Prognostic factors are contained in the surgical stage: type, grade, depth of myometrial invasion and extension to the cervix. Some oncologists prefer the designation based on thirds (inner, mid, outer) of myometrial invasion or distance in $\mathrm{mm}$ from the serosal surface instead of halves of myometrium, and pathologists may report the maximal depth of tumor invasion from the endomyometrial junction and the thickness of the myometrium at that point to be more accurate. The myometrial invasion must be differentiated from carcinomatous extension into pre-existing "tongues" of endometrium penetrating the myometrium or into foci of adenomyosis [13]. Perivascular lymphocytic infiltrates should prompt deeper levels of histological examination, as they may indicate vascular and lymphatic invasion (a marker of poor prognosis).

Endometrioid adenocarcinoma spreads by lymphatic and vascular dissemination, transperitoneal or transtubal seeding, and direct extension to contiguous organs. The later pathway was considered in the presented case, although no cervical involvement was noted at the initial diagnosis. The relative frequency of vaginal metastasis from endometrial carcinoma range from $18 \%$ to $25 \%$ [4], so secondary spread of malignant neoplasms to the vagina is quite common [4]. Metastatic vaginal carcinomas may also originate from the colon, rectum, urinary tract and, more rarely, the breast. The primary tumor is often clinically evident or, as in the reported case, has previously been treated. In our case biopsy allowed the diagnosis, showing a similar histologic aspect with the previous endometrial endometrioid carcinoma.

Ovarian mucinous adenocarcinomas express genetic heterogeneity, probably as a re- 
flection of the progression from benign to malignant neoplasia. Recent studies strongly suggest that in the sequence of malignant transformation from benign to malignant tumors, borderline mucinuos adenocarcinomas, intraepithelial (non-invasive) carcinomas, and expansile invasive carcinomas represent transitional stages of mucinous carcinogenesis [5]. Molecular studies of genetic alterations in mucinous tumors support this hypothesis, finding KRAS mutational activation (noted also in endometrioid carcinoma) as an early event $[8,11]$.

Generally the grading of mucinous carcinomas has not been shown to be predictive of behavior or response to therapy independent of the surgical stage, except microinvasion, as all individual invasive foci are less than $10 \mathrm{~mm}^{2}[5]$.

Malignant peripheral nerve sheath tumors (MPNSTs or synonyms: neurogenic sarcoma, neurofibrosarcoma, malignant schwannoma) are rare neoplasms accounting for about $5 \%$ of malignant tumors of soft tissues. MPNSTs are highly malignant sarcomas that are locally invasive, frequently leading to multiple recurrences and eventual metastatic spread.

Macroscopy shows globoid or fusiform, pseudoencapsulated tumor that is firm to hard in consistency, the cut surface is typically cream colored or grey, with foci of necrosis and haemorrhage that are sometimes extensive. The diameter of about $9 \mathrm{~cm}$ found in the reported case corresponds to the size of more than $5 \mathrm{~cm}$ characteristically noted in the vast majority of MPNSTs [2]. In MPNSTs, tumor cells can show differentiation of any cell types of the nerve sheath, including Schwann cell, perineural fibroblast, or fibroblast, all of which were also observed in the present case.

MPNST may arise from malignant transformation of a neurofibroma, often of the plexiform type and in the setting of neurofibromatosis type 1 (NF1). Only rare examples also develop from conventional schwannomas or ganglioneuromas [2, 3]. Other cases appear de novo or after the irradiation of a neuroblastic tumor (up to $10 \%$ of MPNSTs, with a post-treatment latency of more than 10 years).

In most of the cases microscopy reveals fibrosarcoma-like fasciculated growth as packed, hyperchromatic spindle cells with abundant faintly eosinophilic cytoplasm, also observed in the presented case. Nuclei are elongated, heterochromatic, focally pleomorphic and with inconspicuous nucleoli. Tapered ends of nuclei frequently express a wavy or buckled configuration [3]. Mitoses are easily noticed. Characteristic abrupt alternation between cellular areas and more mixoid areas is typical and were also observed in this case. Up to $10 \%$ of similar cases have a mixoid stroma (mixoid MPNST). Perivascular accentuation or whorling of tumor cells that sometimes extend in the vessel walls and develop thrombosis may also be noted. In some cases they express a uniformity of cells or fascicular aspect that cannot be differentiated on HE from synovial monophasic sarcoma or from fibrosarcoma. A plexiform arrangement may be observed in tumors that appear in childhood.

Histological variants are: epithelioid, glandular, malignant Triton tumor with rhabdomyosarcomatous differentiation and pigmentar (melanotic) or melanotic schwannoma $[2,3]$. Epithelioid types have a multinodular aspect, being composed of nests or cords of 
round epithelioid eosinophilic or amphopilic cells, with vesicular nuclei and proeminent nucleoli. Glandular type is composed of gland-forming epithelium, intestinal-appearing, keratin- and CEA- positive glands, frequently with intra- and extracellular mucin, and neuroendocrine cells with immunoreactivity for chromogranin, somatostatin, and serotonin. Malignant Triton tumor with rhabdomyosarcomatous differentiation frequently expresses association with areas of chondrosarcoma, osteosarcoma or epithelial glands (pluridirectional differentiation) [2,3].

In 50-70 \% of MPNSTs, and also in the reported case, immunohistochemistry shows scattered S-100 positive tumor cells. The explanation is that ultrastructurally, only $50 \%$ of tumors present well- developed schwannian differentiation, while other cases present fibroblastic, perineural or mixed patterns. The majority of MPNSTs are p53 positive, while p27 and p16 are commonly expressed in neurofibroma not in MPNST [3]. Occasionally, there are EMA positive cells, showing perineural differentiation. Proliferation markers, as Ki-67/MIB-1 are positive in 5-65 \% of cases. The immunoreactivity profile in our case presented also Vimentin and NSE positivity (the latter being useful in excluding the diagnosis of liposarcoma), SM-Actin and Desmin negativity.

MPNSTs vary from low grade lesions to high grade tumors, the majority featuring high cellularity, brisk mitotic activity and necrosis (grade III/IV) [3], corresponding to the microscopic aspect found in the presented case. No firm association has been established between histological grade and survival.

The molecular alterations contributing to MPNSTs tumorigenesis are atributed to $p 53$. The $p 53$ tumor suppressor gene is mutated in more than half of human cancers and seems to play an important role in MPNSTs, because deletions and mutations affecting the $p 53$ gene locus, as well as nuclear accumulation of p53 protein, have been identified in these cases. Furthermore, the recent demonstration of homozygous deletions of the CDKN2A gene, together with alterations in the expression of p27Kip1, are additional evidence of an altered cell-cycle control in MPNSTs. Recently, a novel candidate tumor suppressor gene, the PTEN gene (involved also, as mentioned, in endometrial carcinogenesis), also named $M M A C 1$ or TEP1, has been identified on chromosome 10q23.3. In its signaling process of G1 cell-cycle arrest and/or apoptosis, one critical target is the cyclin-dependent kinase inhibitor p27 (KIP1). Recruitment of p27 (KIP1) to cyclin E immunocomplexes correlates to cell-cycle arrest and leads to a reduction in the cyclindependent kinase $2(\mathrm{cdk} 2)$ activity and subsequent decrease in the levels of endogenous phosphorylated retinoblastoma $(\mathrm{RB})$ protein. Because $\mathrm{RB}$ is known to play a central role in the regulation of the cell cycle, functional inactivation due to inappropriate phosphorylation of the $\mathrm{RB}$ protein resulting from deregulation of upstream effectors in the $\mathrm{RB}$ pathway (e.g. p16 inactivation or cyclin overexpression) or due to mutations in the $R b$ gene itself may result in a malignant phenotype [9].

The reported association of malignant epithelial and peripheral nerve sheath tumors is rare and may suggest some similar induction pathways in tumorigenesis, knowing their common altered cell-cycle control, with involvement of PTEN (correlated with p27) and $p 53$ in the genesis of both types of malignancies [7, 9]. 
Although the synchronous tumors of endometrium and ovary were of a relatively early stage and consequently had a favorable prognosis, subsequently occurring implants along the lower genital tract and metachronous MPNST added up to a poor prognosis.

\section{Acknowledgment}

The authors are grateful to Prof. Dr F.T. Bosman (Head of the Institute of Pathology, University of Lausanne, Switzerland) for his expert assistance.

\section{References}

[1] G.J. Beattie, J.R. Livingstone, M.A. Cornbleet, J.F. Smyth and K.M. McLaren: "Mucinous endometrial carcinoma in a patient with previous ovarian endometrioid adenocarcinoma presenting some 18 years after initial bilateral ovariectomy: a case report", Scott. Med. J., Vol. 36(6), (1991), pp. 181-182.

[2] C.D.M. Fletcher (Ed.): Diagnostic Histopathology of Tumors, Vol. 2, Churchill Livingstone, Edinburgh, 2000.

[3] P. Kleiheus and W.K. Cavenee (Eds.): WHO Classification of Tumors, Pathology and Genetics of Tumors of the Nervous System, IARC Press, Lyon, 2000.

[4] R.J. Kurman (Ed.): Blaustein's Pathology of the Female genital Tract, 5th ed., Springer-Verlag, New York, 2002.

[5] K.R. Lee and R.E. Scully: "Mucinous tumors of the ovary: a clinicopathologic study of 196 borderline tumors (of intestinal type) and carcinomas, including an evaluation of 11 cases with „pseudomyxoma peritonei"”, Am. J. Surg. Pathol., Vol. 24, (2000), pp. $1447-1464$.

[6] K.R. Lee and R.H. Young: "The distinction between primary and metastatic mucinous carcinomas of the ovary: gross and histologic findings in 50 cases", Am. J. Surg. Pathol., Vol. 27, (2003), pp. 281-293.

[7] R.L. Levine, C.B. Cargile, M.S. Blazes, B. van Rees, R.J. Kurman and L.H. Ellenson: "PTEN mutations and microsatellite instability in complex atypical hyperplasia, a precursor lesion to uterine endometrioid carcinoma", Cancer Res., Vol. 58, (1998), pp. 3254-3258.

[8] M. Mandai, I. Konishi, H. Kuroda, T. Komatsu, S. Yamamoto, K. Nanbu, K. Matsushita, M. Fukumoto, H. Yamabe and T. Mori: "Heterogenous distribution of K-ras mutated epithelia in mucinous ovarian tumors with special reference to histopathology", Hum. Pathol., Vol. 29, (1998), pp. 34-40.

[9] C. Mawrin, E. Kirches, C. Boltze, K. Dietzmann, A. Roessner and R. SchneiderStock: "Immunohistochemical and molecular analysis of p53, RB, and PTEN in malignant peripheral nerve sheath tumors", Virchows Arch Springer-Verlag, Vol. 440(6), (2002), pp. 610-615.

[10] G.L. Mutter: "Pten, a protean tumor suppressor", Am. J. Pathol., Vol. 158, (2001), pp. 1895-1898. 
[11] G.L. Mutter, T.A. Ince, J.P. Baak, G.A. Kust, X.P. Zhou and C. Eng: "Molecular identification of latent precancers in histologically normal endometrium", Cancer Res., Vol. 61, (2001), pp. 4311-4314.

[12] R.R. Raju, W.R. Hart, D.K. Magnuson, J.R. Reid and D.G. Rogers: "Genital tract tumors in Proteus syndrome: report of a case of bilateral paraovarian endometrioid cystic tumors of borderline malignancy and review of the literature", Mod. Pathol., Vol. 15(2), (2002), pp. 172-180.

[13] F.A. Tavassoli and P. Deville (Eds.): WHO Classification of Tumors of the Breast and Female Genital Organs, IARC Press, Lyon, 2003.

[14] J.D. Woodruff and C.G. Julian "Multiple malignancy in the upper genital canal", Am. J. Obstet. Gynecol., Vol. 103, (1969), pp. 810-822.

[15] R. Zaino, C. Whitney, M.F. Brady, K. DeGeest, R.A. Burger and R.E. Buller: "Simultaneously detected endometrial and ovarian carcinomas-a prospective clinicopathologic study of 74 cases: a gynecologic oncology group study", Gynecol. Oncol., Vol. 83(2), (2001), pp. 355-362. 